\title{
Multifactorial inhibition of lactobacilli against the respiratory tract pathogen Moraxella
} catarrhalis

\author{
M.F.L. van den Broek ${ }^{1}$, I. De Boeck ${ }^{1}$, I.J.J. Claes ${ }^{1}$, V. Nizet $^{2,3}$ and S. Lebeer ${ }^{1 *}$ \\ ${ }^{1}$ Department of Bioscience Engineering, Research Group Environmental Ecology and Applied Microbiology, University of \\ Antwerp, Groenenborgerlaan 171, 2020 Antwerp, Belgium; ${ }^{2}$ Department of Pediatrics, Division of Host-Microbe Systems \\ and Therapeutics, University of California, San Diego, 9500 Gilman Drive, MC 0760, La Jolla, CA 92093-0760, USA; \\ ${ }^{3}$ Skaggs School of Pharmacy and Pharmaceutical Sciences, University of California, San Diego, 9500 Gilman Drive, MC \\ 0687, La Jolla, CA 92093-0760,USA; sarah.lebeer@uantwerpen.be
}

Received: 12 July 2017 / Accepted: 2 December 2017

(c) 2018 Wageningen Academic Publishers

OPEN ACCESS CC)

\begin{abstract}
Probiotics, mainly lactic acid bacteria (LAB), are widely focused on gastrointestinal applications. However, recent microbiome studies indicate that LAB can be endogenous members of other human body sites such as the upper respiratory tract (URT). Interestingly, DNA-based microbiome research suggests an inverse correlation between the presence of LAB and the occurrence of potential pathogens, such as Moraxella catarrhalis, an important URT pathogen linked to otitis media, sinusitis and chronic obstructive pulmonary disease. However, a direct interaction between these microbes has not been explored in detail. This study investigated the direct antipathogenic effects of Lactobacillus species, including several well-documented probiotic strains, on M. catarrhalis using agar-based assays, time course analysis, biofilm assays and minimal inhibitory concentration (MIC) testing. These assays were performed using spent culture supernatans (SCS) at two pHs (4.3 and 7) and D- and/or L-lactic acid at three pHs (2, 4 and 7). In addition, cell line assays for adhesion competition and immunomodulation were used to substantiate the inhibitory effect of lactobacilli against $M$. catarrhalis. A proportion of Lactobacillus strains, including the model probiotic Lactobacillus rhamnosus GG, showed a strong and direct activity against $M$. catarrhalis. Screening of the activity of the SCS after different treatments demonstrated that lactic acid has an important antimicrobial activity against this pathogen - at least in vitro - with mean MIC values for D- and L-lactic acid varying between 0.5 and 27 $\mathrm{g} / \mathrm{l}$ depending on the $\mathrm{pH}$. Furthermore, L. rhamnosus $\mathrm{GG}$ also decreased the adhesion of $M$. catarrhalis to human airway epithelial Calu- 3 cells with more than $50 \%$, and the expression of mucin MUC5AC, pro-inflammatory cytokines interleukin (IL)-8, IL-1 $\beta$, and tumor necrosis factor- $\alpha$ at least 1.2 fold. This study suggests that several lactobacilli and their key metabolite lactic acid are possible candidates for probiotic therapeutic interventions against URT infections.
\end{abstract}

Keywords: probiotics, upper respiratory tract, Lactobacillus rhamnosus GG

\section{Introduction}

Moraxella catarrhalis is a Gram-negative pathogen implicated in many airway diseases, such as $15-20 \%$ of acute otitis media (OM) infections, $20 \%$ of acute sinusitis episodes in children and $10 \%$ of the chronic obstructive pulmonary disease exerbations in adults (Murphy et al., 2009; Parameswaran et al., 2009; Rovers et al., 2004). Interestingly, while the colonisation rate is low in adults
(1-5\%), M. catarrhalis is a common coloniser of the infant nasopharynx (66-100\%) (Murphy et al., 2009). To treat upper respiratory tract (URT) infections, antibiotics are still considered as standard therapy, while they have many side-effects including the induction of antibiotic resistance (Goossens et al., 2005). Indeed, a high proportion of $M$. catarrhalis strains are now resistant to beta-lactam antibiotics such as penicillin and amoxicillin (Harrison et al., 2009). Moreover, antibiotics disturb the endogenous 
beneficial microbiota, especially when taken orally. Elimination of key commensal bacteria can facilitate pathogen colonisation in the gastro-intestinal tract and nasopharynx (Tagg and Dierksen, 2003), resulting in unwanted side-effects such as diarrhea and increased susceptibility to subsequent infections. In addition to pathogen restriction, an intact healthy microbiota plays a key role in the education and function of the immune system (Bogaert et al., 2011; Pettigrew et al., 2012; Stearns et al., 2015). For all these reasons, an urgent need exists for alternatives to classical antibiotic therapy for infections with Moraxella and other URT pathogens.

In the gastro-intestinal and vaginal tract, probiotic lactobacilli have long been explored as alternatives for antibiotics. Interestingly, several recent microbiome studies have indicated that genera of lactic acid bacteria (LAB) such as Lactobacillus and Lactococcus can also be normal inhabitants of the URT, in variable relative abundances (Bogaert et al., 2011; Pettigrew et al., 2012; Stearns et al., 2015). In children, a greater abundance of taxa such as Lactococcus and Dolosigranulum (both LABs) appear to be inversely correlated with Moraxella and the risk of developing acute OM (Biesbroek et al., 2014; Pettigrew et al., 2012). Bogaert and colleagues (2011) observed a marked increase in the absolute incidence of (Brevi)bacillus and Lactobacillus species colonisation in summer (96\%) compared to winter (10\%) season, the latter of which has the highest prevalence of URT infections. Stearns and colleagues (2015) reported that Lactobacillus strains were present in the nasopharyngeal samples of all 51 healthy children tested with a relative abundance varying between 0.0026 and $5.62 \%$, making it the $13^{\text {th }}$ most abundant species in that niche. Among the 19 healthy adults included in their study, lactobacilli were even more prevalent, ranked at the $11^{\text {th }}$ place among commensal species with an abundance varying between 0.0057 and $13.29 \%$. However, these DNAbased microbiome studies have not yet been backed up with mechanistic studies on the direct interactions between LAB and the corresponding URT pathogens. Yet, pilot studies have already shown that the administration of certain $\mathrm{LAB}$ could mediate a reduction in the incidence of $\mathrm{OM}$ in children (Hatakka et al., 2007; Niittynen et al., 2012; Rautava et al., 2009). However, until now, most of these probiotics were applied orally in liquid formulas for immune boosting effects, with little attention to the possibility of direct inhibition of URT pathogens by the applied LAB.

In this study, the direct antimicrobial activities of various Lactobacillus strains, including several well-documented probiotic strains, were explored against $M$. catarrhalis. The analyses were conducted using optimised assays allowing detailed insights in 1:1 competitions between potential probiotic and pathogen.

\section{Materials and methods}

\section{Bacterial strains and growth conditions}

Lactobacillus strains (Table 1 ) were grown at $37^{\circ} \mathrm{C}$ in de Man, Rogosa and Sharpe (MRS) medium (BD Difco, Erembodegem, Belgium). M. catarrhalis ATCC25238 was inoculated in Mueller Hinton (MH) (LabM Limited, Heywood, UK) broth and cultured aerobically at $37^{\circ} \mathrm{C}$. Solid media contained 1.5\% (w/v) agar. For antimicrobial assays, the agar was enriched with glucose (Sigma Aldrich, Steinheim, Germany) to a final concentration of $5 \mathrm{~g} / \mathrm{l}$.

\section{Cell culture}

The human human airway epithelial cell line Calu-3 ATCC ${ }^{\bullet}$ HTB-55TM (purchased from ATCC, Molsheim Cedex, France) was cultured in $75 \mathrm{~cm}^{2}$ flasks containing $20 \mathrm{ml}$ Minimum Essential Medium (MEM) (Life Technologies, Erembodegem, Belgium) supplemented with heat inactivated fetal bovine serum (FBS) (Thermo Fischer, Asse, Belgium) and penicillin-streptomycin (100 U/ml) (Life Technologies) and maintained in a humidified $5 \% \mathrm{CO}_{2}$ incubator at $37^{\circ} \mathrm{C}$. The culture medium was changed every 3-4 days and the cells were passaged weekly at a 1:2 split ratio using a $0.25 \%$ trypsin-EDTA solution (Life Technologies). For adhesion and immunomodulation experiments, $0.5 \mathrm{ml}$ of Calu-3 cells were seeded in 24-well culture plates at a density of $1.85 \times 10^{6}$ cells $/ \mathrm{ml}$. Approximately a week after seeding, confluent monolayers were obtained.

\section{Preparation of spent culture supernatant of lactobacilli}

Lactobacilli were incubated for $19 \mathrm{~h}$ (corresponding to a final concentration of $\pm 2 \times 10^{9} \mathrm{cfu} / \mathrm{ml}$ ) and spent culture supernatant (SCS) was obtained by centrifugation for $15 \mathrm{~min}$ at 4,000 rpm at $4{ }^{\circ} \mathrm{C}$. Afterwards, the SCS was filter sterilised $(0.20 \mu \mathrm{m}$ cellulose acetate, VWR, Leuven, Belgium) and the pH (Mettler-Toledo AG, Zaventem, Belgium) and the concentration of D- and L-lactic acid (Roche Yellow Line, R-Biopharm AG, Darmstadt, Germany) were measured. To gain information on the nature of the active antimicrobial molecules produced by the lactobacilli, the SCSs of the different lactobacilli were treated by different methods: (1) heating at $70{ }^{\circ} \mathrm{C}$ for $30 \mathrm{~min}$; (2) treatment with proteinase $\mathrm{K}(50 \mu \mathrm{g} / \mathrm{ml})$ (Sigma-Aldrich, St. Louis, MO, USA) and incubation for $60 \mathrm{~min}$ at $37^{\circ} \mathrm{C}$; and (3) neutralisation to pH 7 by using $\mathrm{NaOH}$.

\section{Growth characteristics of lactobacilli}

Growth was measured using Synergy HTX multimode plate reader (BioTek, Winooski VT, USA) by measuring optical density (OD) at $595 \mathrm{~nm}$. 
Table 1. Bacterial strains used in this study.

\begin{tabular}{|c|c|c|c|}
\hline Species & Strain & Relevant genotype or description & Reference and/or source \\
\hline \multicolumn{4}{|l|}{ Lactobacilli } \\
\hline Lactobacillus casei & ATCC334 & $\begin{array}{l}\text { Single colony isolate obtained from a stock culture of ATCC334. } \\
\text { Originally isolated from emmental cheese }\end{array}$ & ATCC \\
\hline L. casei & Shirota & $\begin{array}{l}\text { Single colony isolate obtained from a commercially available fermented } \\
\text { drink containing } L \text {. casei Shirota }\left(\text { Yakult }^{\mathrm{R}}\right) \text {, confirmed by sequencing }\end{array}$ & $\begin{array}{l}\text { Commercial probiotic } \\
\text { product }\end{array}$ \\
\hline L. casei & DN-114001 & $\begin{array}{l}\text { Single colony isolate obtained in our lab from a commercially available } \\
\text { fermented drink (Actimel }{ }^{\circledR} \text { ) containing L. casei DN-114001, confirmed } \\
\text { by sequencing }\end{array}$ & Lebeer et al., 2007 \\
\hline Lactobacillus parabuchneri & NM63-3 & Single colony isolate from spontaneously fermented carrot juice & Lab collection \\
\hline Lactobacillus paracasei & LMG12586 & $\begin{array}{l}\text { Single colony isolate obtained from a stock culture of LMG12586. } \\
\text { Originally isolated from cheese }\end{array}$ & BCCM/LMG \\
\hline Lactobacillus plantarum & LMG1284 & $\begin{array}{l}\text { Single colony isolate from L. plantarum ATCC8014 or LMG1284. Origin } \\
\text { unknown }\end{array}$ & BCCM/LMG \\
\hline L. plantarum & CMPG5300 & Single colony isolate. Originally isolated from the vaginal cavity & Malik et al., 2014 \\
\hline L. plantarum & 5057 & $\begin{array}{l}\text { Single colony isolate of } L \text {. plantarum } 5057 \text {. Originally isolated from } \\
\text { maize silage }\end{array}$ & Danielsen, 2002 \\
\hline L. plantarum & WCFS1 & $\begin{array}{l}\text { Single colony isolate of } L \text {. plantarum WCFS1. Originally isolated from } \\
\text { human saliva }\end{array}$ & Kleerebezem et al., 2003 \\
\hline Lactobacillus reuteri & $\mathrm{RC}-14$ & $\begin{array}{l}\text { Single colony isolate from a commercially available probiotic supplement } \\
\text { containing } L \text {. reuteri } R C-14 \text {, confirmed by sequencing }\end{array}$ & $\begin{array}{l}\text { Commercial probiotic } \\
\text { product }\end{array}$ \\
\hline Lactobacillus rhamnosus & GR-1 & $\begin{array}{l}\text { Single colony isolate obtained from a commercially available probiotic } \\
\text { supplement containing L. rhamnosus GR-1. Originally isolated from } \\
\text { the female urethra }\end{array}$ & $\begin{array}{l}\text { Chan et al., 1984, 1985; } \\
\text { Reid and Bruce, 2001; } \\
\text { Reid and Reid, 1999, } \\
\text { ATCC }\end{array}$ \\
\hline L. rhamnosus & GG & $\begin{array}{l}\text { Single colony isolate Wild type strain, originally isolated from human } \\
\text { faeces }\end{array}$ & Kankainen et al., 2009 \\
\hline \multicolumn{4}{|l|}{ Pathogens/control bacteria } \\
\hline Haemophilus influenzae & ATCC49247 & $\begin{array}{l}\text { Single colony isolated from expectorated sputum from a } 76 \text { year-old } \\
\text { male with pneumonia }\end{array}$ & ATCC \\
\hline Moraxella catarrhalis & ATCC25238 & Type strain, originally isolated from the nasal cavity & ATCC \\
\hline Streptococcus pneumoniae & ATCC49619 & Single colony isolated from sputum of 75 -year-old male & ATCC \\
\hline Escherichia coli & LMG2093 & $\begin{array}{l}\text { Single colony isolate obtained from a stock culture of LMG2093. Origin } \\
\text { unknown }\end{array}$ & BCCM/LMG \\
\hline
\end{tabular}

\section{Spot antimicrobial assays with live lactobacilli}

The antimicrobial activity of live lactobacilli against the $M$. catarrhalis was explored by standard antimicrobial tests. The antimicrobial activity of Lactobacillus cultures was explored by modified spot assays (Schillinger and Lücke, 1989). Briefly, $2 \mu \mathrm{l}$ of each Lactobacillus culture was spotted on a standard agar plate $(1.5 \% \mathrm{w} / \mathrm{v})$ containing medium of the pathogen supplemented to a final concentration of 5 $\mathrm{g} / \mathrm{l}$ glucose and a spot of another bacterium was added as negative control. These plates were incubated for $48 \mathrm{~h}$ at $37^{\circ} \mathrm{C}$. Ca. $10^{6} \mathrm{cfu} / \mathrm{ml}$ of the overnight pathogen culture were added to $7 \mathrm{ml}$ of soft agar $(0.5 \% \mathrm{w} / \mathrm{v})$ and poured over the plates with Lactobacillus spots. The plates were incubated overnight at $37^{\circ} \mathrm{C}$, after which time the inhibition zones were measured.

\section{Radial diffusion antimicrobial assay for SCS of lactobacilli}

The antimicrobial activity of SCS was investigated with a modified protocol based on previously described competition assays between lactobacilli and gastrointestinal pathogens (Lie et al., 1997). Approximately $10^{6} \mathrm{cfu} / \mathrm{ml}$ of $M$. catarrhalis was added to $60 \mathrm{ml}$ of $1.5 \%$ $(\mathrm{w} / \mathrm{v})$ agar and poured in a square agar plate. After the plates dried, wholes of $0.4 \mathrm{~cm}$ diameter were made in the agar and $30 \mu \mathrm{l}$ of SCS of lactobacilli was added. The plates were incubated overnight and the inhibition zones were measured. 0.1\% hexetidine (Famar Orléans, Orléans, France) and sterile MRS medium brought to $\mathrm{pH} 4.3$ were used as a positive and negative control, respectively. 


\section{Time-course analysis of the antimicrobial activity of spent culture supernatans}

A time-course analysis of the growth inhibition in suspension was performed as described previously (De Keersmaecker et al., 2006) with minor modifications. Briefly, $190 \mu \mathrm{l}$ of a diluted overnight culture of $M$. catarrhalis (ca. $10^{5} \mathrm{cfu} / \mathrm{ml}$ ) was added to the wells of a microplate supplemented with $10 \mu \mathrm{l} \mathrm{SCS}$ of lactobacilli to get a total volume of $200 \mu \mathrm{l} .10 \mu \mathrm{l} 0.1 \%$ hexetidine and $10 \mu \mathrm{l}$ MRS medium at $\mathrm{pH} 4.3$ were used as a positive and negative control, respectively. Bacteria were grown and the OD was measured at $600 \mathrm{~nm}$ each $30 \mathrm{~min}$ using a Synergy HTX multi-mode reader. Each test was measured at least in triplicate and the average OD was calculated.

\section{Minimal inhibitory concentration assay}

The minimal inhibitory concentration (MIC) values were determined based on the procedure described earlier (Nizet et al., 2001). M. catarrhalis was grown in Mueller Hinton medium as described above. A 96-well plate was filled to get a total volume of $100 \mu \mathrm{l}$ with a final concentration of $5 \times 10^{5} \mathrm{cfu} / \mathrm{ml}$ of the pathogen combined with an adequate concentration of the active molecule. The microplate was incubated overnight at $37^{\circ} \mathrm{C}$ and the $\mathrm{OD}$ at $600 \mathrm{~nm}$ was measured.

\section{Biofilm assays}

Biofilm formation of the pathogens in the presence of SCS of the lactobacilli was monitored using static biofilm assays. For $M$. catarrhalis, biofilms were grown as described by Pearson and Hansen (2007) with minor modifications. An overnight culture of $M$. catarrhalis was diluted 1:100 $\left( \pm 10^{5}\right.$ $\mathrm{cfu} / \mathrm{ml}$ ) in Brain Heart Infusion broth (Difco, BD, Franklin Lakes, NJ, USA). A volume of $190 \mu \mathrm{l}$ of this suspension was loaded into a 96-well microplate and incubated overnight at $37^{\circ} \mathrm{C}$. To test the antimicrobial activity of the SCS, $10 \mu \mathrm{l}$ portions of SCS were loaded into the 96-well microplate. $10 \mu \mathrm{l} 0.1 \%$ hexetidine and $10 \mu \mathrm{l}$ MRS medium at $\mathrm{pH} 4.3$ were used as a positive and negative, respectively. After 19 $h$, the broth was removed from each well and replaced by $200 \mu \mathrm{l}$ phosphate buffered saline (PBS) plus $10 \mu \mathrm{l}$ of $0.7 \%$ $(\mathrm{w} / \mathrm{v})$ crystal violet. After $15 \mathrm{~min}$ at room temperature, the wells were emptied and washed three times with deionised water. A $200-\mu \mathrm{l}$ volume of $95 \%$ ethanol was added to each well, and the plate was shaken gently for $15 \mathrm{~min}$. A $150 \mu \mathrm{l}$ portion of this ethanol solution was then transferred to a new 96-well plate, and the absorbance at $570 \mathrm{~nm}$ was measured using a Synergy HTX multi-mode reader.

\section{Adhesion competition assays}

The influence of lactobacilli on the adherence of $M$. catarrhalis to human airway epithelial Calu-3 cells was investigated as described previously (Malik et al., 2013) with minor modifications. Competition tests were carried out by adding a volume of $0.5 \mathrm{ml}$ containing $M$. catarrhalis $\left(5 \times 10^{7}\right.$ or $\left.5 \times 10^{6} \mathrm{cfu}\right)$ and Lactobacillus rhamnosus GG $\left(5 \times 10^{7} \mathrm{cfu}\right)$ to a tissue culture 24 -well plate containing confluent monolayers of epithelial cells, which were allowed to incubate at $37^{\circ} \mathrm{C}$ for $1 \mathrm{~h}$ to mediate adherence. After incubation, the cells were washed three times with PBS $\left(37^{\circ} \mathrm{C}\right)$ to remove all nonadhering cells and the number of adhering pathogenic cells to the Calu-3 cells was determined by macrodilution method on appropriate agar. Each condition was carried out at least in triplicate.

\section{Induction of cytokine gene expression in Calu-3 epithelial cells}

The cytokine response after co-incubation of Calu-3 cells with $M$. catarrhalis and L. rhamnosus GG was determined as follows. The bacterial strains were first grown overnight and subsequently centrifuged at $2,000 \times g$ at $4{ }^{\circ} \mathrm{C}$ for $10 \mathrm{~min}$, washed with PBS and resuspended in MEM medium. $M$. catarrhalis $\left(5 \times 10^{7} \mathrm{cfu}\right)$ was added to the Calu-3 epithelial cells, alone or in presence of L. rhamnosus GG $\left(5 \times 10^{7}\right.$ $\mathrm{cfu}$ ). After $4 \mathrm{~h}$ of incubation, the cells were rinsed thrice with PBS. Next, total RNA was extracted from the Calu-3 cells with RNeasy isolation kit (Qiagen, Hilden, Germany) following the manufacturer's protocol. A constant amount of $1 \mu \mathrm{g}$ of total RNA was used for oligo-(dT)-primed cDNA synthesis using ReadyScript ${ }^{\oplus}$ reverse transcriptase (Sigma Aldrich). The cytokine gene expression was measurement by quantitative real time polymerase chain reaction (qRTPCR).

\section{qRT-PCR}

Expression of interleukin (IL)-8, IL-1 $\beta$, mucin MUC5AC and tumour necrosis factor- $\alpha$ (TNF- $\alpha$ ) was analysed using Power SYBR Green PCR Master Mix (Applied Biosystems, Foster City, CA, USA) according to manufacturer's instructions. Data were quantified with the $\Delta \Delta \mathrm{Ct}$ method, relative to the reference genes Cytochrome c1 (CYC1) and ATP synthase, $\mathrm{H}+$ transporting, mitochondrial F1 complex, beta polypeptide (ATP5B). Primer sequences can be found in Table 2. Minimum Information for Publication of Quantitative Real-Time PCR Experiments (MIQE) guidelines were strictly followed (Bustin et al., 2009).

\section{Statistics}

Data are represented as mean values \pm standard deviation. One-way ANOVA and multiple t-tests were used to determine statistical significance in GraphPad Prism (La 
Table 2. Primers used for qRT-PCR.

\begin{tabular}{ll} 
Primer $^{1}$ & Oligonucleotide sequence (5'-3') \\
CYC1 (F) & CATGTCCCAGATAGCCAAGGA \\
CYC1 (R) & CTTGTGCCGCTTTATGGTGTAG \\
ATP5B (F) & GCAGGAAAGATTACCACTACCAAG \\
ATP5B (R) & TGGTAGCATCCAAATGGGCAA \\
IL-1 1 (F) & TTGCTCAAGTGTCTGAAGCAGC \\
IL-1 $(\mathrm{R})$ & CAAGTCATCCTCATTGCCACTG \\
IL-8 $(F)$ & TGGCAGCCTTCCTGATTTCT \\
IL-8 (R) & TTAGCACTCCTTGGCAAAACTG \\
TNF- $\alpha(F)$ & CCTCTGATGGCACCACCAG \\
TNF- $\alpha(R)$ & TCTTCTCGAACCCCGAGTGA \\
MUC5AC (F) & GGGACTTCTCCTACCAAT \\
MUC5AC (R) & TATATGGTGGATCCTGCAGGGTAG \\
\hline${ }^{1} F=$ forward; $R=$ reverse.
\end{tabular}

Jolla, CA, USA). The $P$-value was corrected for multiple comparisons using the Hilm-sidak method in GraphPad Prism. Differences were considered statistically significant at $P<0.05$.

\section{Results}

\section{A proportion of Lactobacillus strains shows antimicrobial activity against $M$. catarrhalis}

The direct interaction between living lactobacilli and $M$. catarhalis was tested via spot assays. In these assays, all lactobacilli tested demonstrated a strong activity against $M$. catarrhalis. Interestingly, several well-known Lactobacillus strains, such as L. rhamnosus GG, Lactobacillus casei Shirota, L. casei DN-114001, Lactobacillus plantarum LMG1284 and L. plantarum WCFS1 were each very active with clear inhibition zones in the range of the positive control $0.1 \%$ hexetidine, a common oral antiseptic agent for oropharyngeal infections (Table 3). As a control, nonLactobacillus microbial species such as Escherichia coli, Haemophilus influenzae and Streptococcus pneumoniae, and the pathogen itself were also included, but expressed no antimicrobial activity against $M$. catarrhalis.

Subsequently, the activities of the secreted metabolites of the Lactobacillus strains were explored by testing the antimicrobial activity of their respective SCS in radial diffusion assays. The SCS of almost all Lactobacillus strains tested showed a strong activity with inhibition zones larger than $0.5 \mathrm{~cm}$ (Table 3).
Table 3. Agar-based antimicrobial screening of the interaction between lactobacilli and Moraxella catarrhalis. ${ }^{1}$

\begin{tabular}{|c|c|c|c|}
\hline Species & Strain & $\begin{array}{l}\text { Spot } \\
\text { assay }^{2}\end{array}$ & $\begin{array}{l}\text { Radial } \\
\text { diffusion } \\
\text { assay }^{2}\end{array}$ \\
\hline Lactobacillus casei & АТСС334 & +++ & ++ \\
\hline L. casei & Shirota & +++ & +++ \\
\hline L. casei & DN-114001 & +++ & +++ \\
\hline Lactobacillus parabuchneri & NM63-3 & +++ & + \\
\hline Lactobacillus paracasei & LMG12586 & +++ & ++ \\
\hline Lactobacillus plantarum & LMG1284 & +++ & +++ \\
\hline L. plantarum & CMPG5300 & +++ & +++ \\
\hline L. plantarum & 5057 & +++ & +++ \\
\hline L. plantarum & WCFS1 & +++ & +++ \\
\hline Lactobacillus reuteri & $\mathrm{RC}-14$ & +++ & +++ \\
\hline Lactobacillus rhamnosus & GR-1 & +++ & +++ \\
\hline L. rhamnosus & GG & +++ & +++ \\
\hline Escherichia coli & LMG2093 & + & - \\
\hline Streptococcus pneumoniae & ATCC49619 & - & - \\
\hline Moraxella catarrhalis & ATCC25238 & - & - \\
\hline Haemophilus influenzae & ATCC49247 & - & - \\
\hline $0.1 \%$ hexetidine & & n.a. & +++ \\
\hline Plain MRS ${ }^{3} \mathrm{pH} 4.3$ & & n.a. & - \\
\hline \multicolumn{4}{|c|}{$\begin{array}{l}1 \text { Inhibition zones of various Lactobacillus strains against } M . \text { catarrhalis } \\
\text { tested in two different assays. } \\
{ }^{2}-=\text { no inhibition; + = 0-0.2 cm inhibition; ++ =0.2-0.5 cm inhibition; +++ } \\
=>0.5 \mathrm{~cm} \text { : inhibition; n.a. = not applicable/available. } \\
{ }^{3} \text { MRS = de Man, Rogosa and Sharpe medium. }\end{array}$} \\
\hline
\end{tabular}

\section{Major antimicrobial compound of $L$. rhamnosus GG against $M$. catarrhalis is $\mathrm{pH}-$ dependent, heat-stable, proteinase K-resistant}

To explore this antimicrobial activity of the SCS further, we performed inhibition experiments with the SCS of different Lactobacillus strains, and monitored the growth of $M$. catarrhalis for $72 \mathrm{~h}$. A prominent strain-specific effect of Lactobacillus SCS and its metabolites against $M$. catarrhalis was observed (Figure 1A). Interestingly, addition of the SCS of the model probiotic strain L. rhamnosus GG inhibited the growth of Moraxella for $72 \mathrm{~h}$ to the same extent as the positive control $(0.1 \%$ hexetidine). This activity was also observed for L. rhamnosus GR-1, L. plantarum CMPG5300 and L. casei ATCC334 but not for slower growing strains exemplified here by L. parabuchneri NM 63-3 (Figure 1A). The negative control, i.e. non-inoculated MRS medium brought to $\mathrm{pH} 4.3$, only induced a small delay in growth of $M$. catarrhalis, indicating that the effect is not merely $\mathrm{pH}$ related. Subsequent survival and plate counting assays indicated that the effect of SCS on the growth of $M$. catarrhalis was bacteriostatic. 

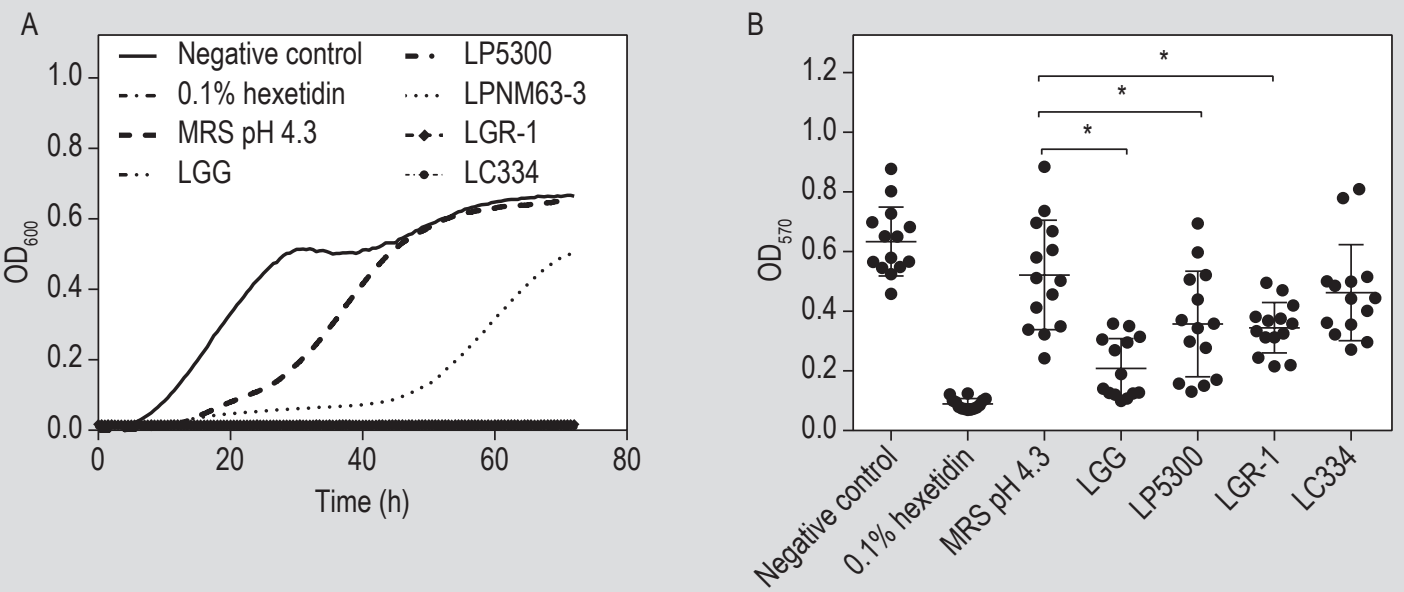

Figure 1. (A) Effect of Lactobacillus spent culture supernatant (SCS) against growth of Moraxella catarrhalis in suspension. Noninoculated MRS medium and $0.1 \%$ hexetidine were used as negative and positive control, respectively. Graphs of LGG, LP5300, LGR-1 and LC334 are overlapping since they do not show growth. (B) The effect of SCS of different Lactobacillus strains on the growth of $M$. catarrhalis as biofilms for $19 \mathrm{~h}$ measured by crystal violet staining $\left(\mathrm{OD}_{570}\right) \cdot{ }^{*} P<0.05$.

Since M. catarrhalis often resides within biofilms in infected patients (Perez et al., 2014), we explored the capacity of SCS of lactobacilli to prevent biofilm formation. As shown in Figure 1B, SCS of L. rhamnosus GG could reduce the biofilm formation of $M$. catarrhalis up to $70 \%$ under the tested conditions. Various other Lactobacillus strains tested such as L. rhamnosus GR-1, Lactobacillus reuteri RC-14, L. casei DN-114001, L. casei Shirota and L. plantarum CMPG5300 also showed the potential to reduce biofilm formation, but their activity appeared less reproducible. For $L$. casei ATCC334, we could not observe a significant reduction under the tested conditions (Figure 1B).

We subsequently aimed to identify the active antimicrobial molecules in the SCS of the lactobacilli, by inactivating or removing different classes of active molecules (acids and proteins). We focused on SCS of L. rhamnosus GG as our model probiotic strain, because this SCS was most active in the experiments described above. The radial diffusion antimicrobial assay showed the antimicrobial activity of SCS of $L$. rhamnosus GG to be $\mathrm{pH}$-dependent, resistant to proteinase $\mathrm{K}$, and heat-stable, since the inhibition zones only disappeared after neutralising the $\mathrm{pH}$ (Figure 2A). In the time-course analysis, the antimicrobial activity disappeared when the SCS was neutralised to $\mathrm{pH} 7$, both when SCS was added in the beginning of the experiment or after overnight incubation of $M$. catarrhalis $(20 \mathrm{~h})$. In contrast, SCS treated with heat or proteinase $\mathrm{K}$ maintained its antimicrobial activity (Figure 2B). Additionally, the antibiofilm activity of the SCS was clearly decreased when the $\mathrm{pH}$ was neutralised. However, merely $\mathrm{pH}$ reduction of MRS medium brought to $\mathrm{pH} 4.3$ by HCl did not significantly affect biofilm formation. Heat and proteinase K-treatment also did not significantly affect the antibiofilm activity (Figure 2C).

\section{Lactic acid is an important antimicrobial compound against $M$. catarrhalis}

Since the antimicrobial activity of lactic acid against certain gastro-intestinal Gram-negative pathogens has been previously documented to be $\mathrm{pH}$-dependent (De Keersmaecker et al., 2006) and since the above-described experiments clearly point to acids as active effector molecules, we subsequently explored the role of lactic acid in the inhibition of the Gram-negative pathogen M. catarrhalis. First, we measured the total amount of lactic acid produced in SCS after overnight growth under standard conditions in MRS medium. This amount differed clearly between the various Lactobacillus strains tested as well as the ratio of D- and L-lactic acid. All L. plantarum strains tested produced almost similar amounts of D- and L-lactic acid, while the other Lactobacillus strains tested appeared to produce almost exclusively L-lactic acid (Figure $3 \mathrm{~A})$. A high $\mathrm{pH}$ and low concentration of LA were linked to decreased activity against $M$. catarrhalis.

To confirm the antimicrobial activity of D- and L-lactic acid, MIC assays were performed against $M$. catarrhalis. Both D- and L-lactic acid showed a strong activity against M. catarrhalis with an MIC of ca. $0.5 \mathrm{~g} / \mathrm{l}$ (Figure 3B). Interestingly, D- and L-lactic acid were almost 10-fold less active when they were dissolved in a neutral solution, confirming the $\mathrm{pH}$-dependent activity of lactic acid.

Other known more indirect antipathogenic activities of probiotics are competitive exclusion and immunomodulation. Here, we could show that L. rhamnosus GG also inhibited the adhesion of different concentrations of M. catarrhalis to Calu-3 cells (Figure 4A). Addition of $L$. 


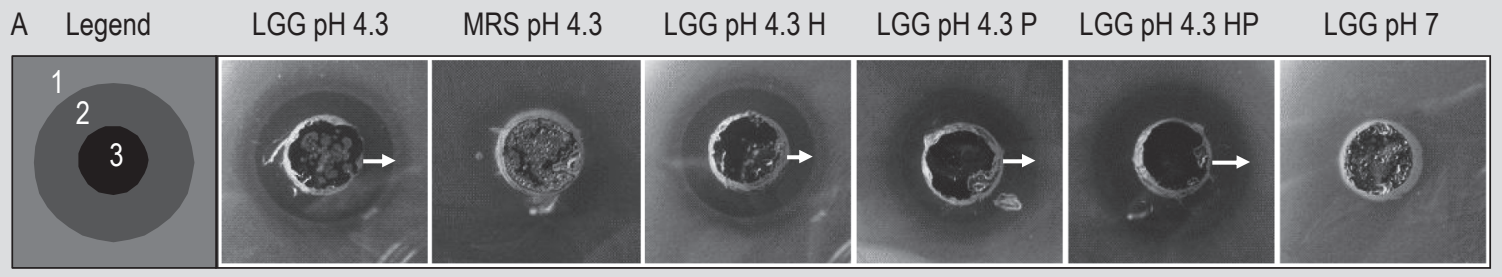

B

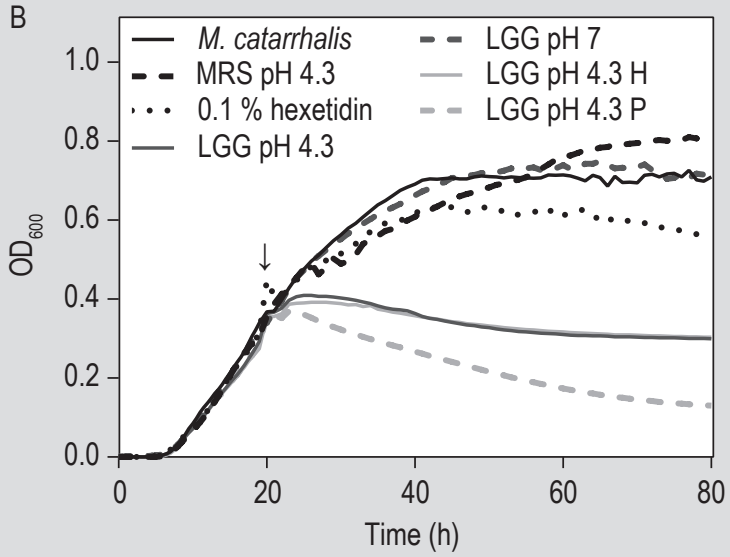

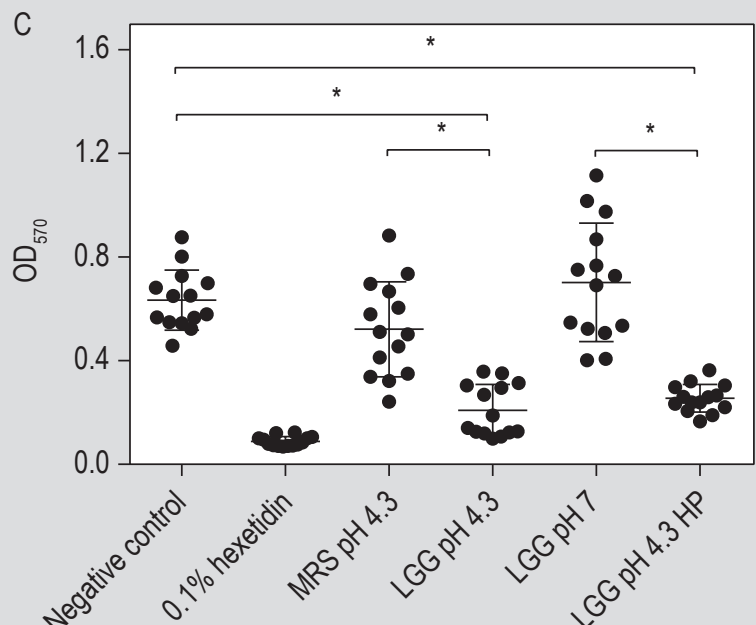

Figure 2. Impact of various treatments on the antimicrobial and antibiofilm effect of spent culture supernatant of Lactobacillus rhamnosus GG (LGG) against Moraxella catarrhalis. (A) Radial-diffusion assay with spent culture supernatant (SCS). 1 = growth pathogen; 2 = inhibition zone; $3=$ well. (B) Time course analysis of $M$. catarrhalis. The arrow indicates the addition of SCS after $20 \mathrm{~h}$ incubation of $M$. catarrhalis. Non-inoculated MRS medium brought to $\mathrm{pH} 4.3$ and $0.1 \%$ hexetidine served as negative and positive control, respectively. (C) Biofilm formation of $M$. catarrhalis. Treated LGG-SCS was added. H = LGG SCS treated with heat; $P=$ LGG SCS treated with proteinase K; HP = LGG-SCS treated with heat and proteinase $K{ }^{*}{ }^{*} P<0.05$.
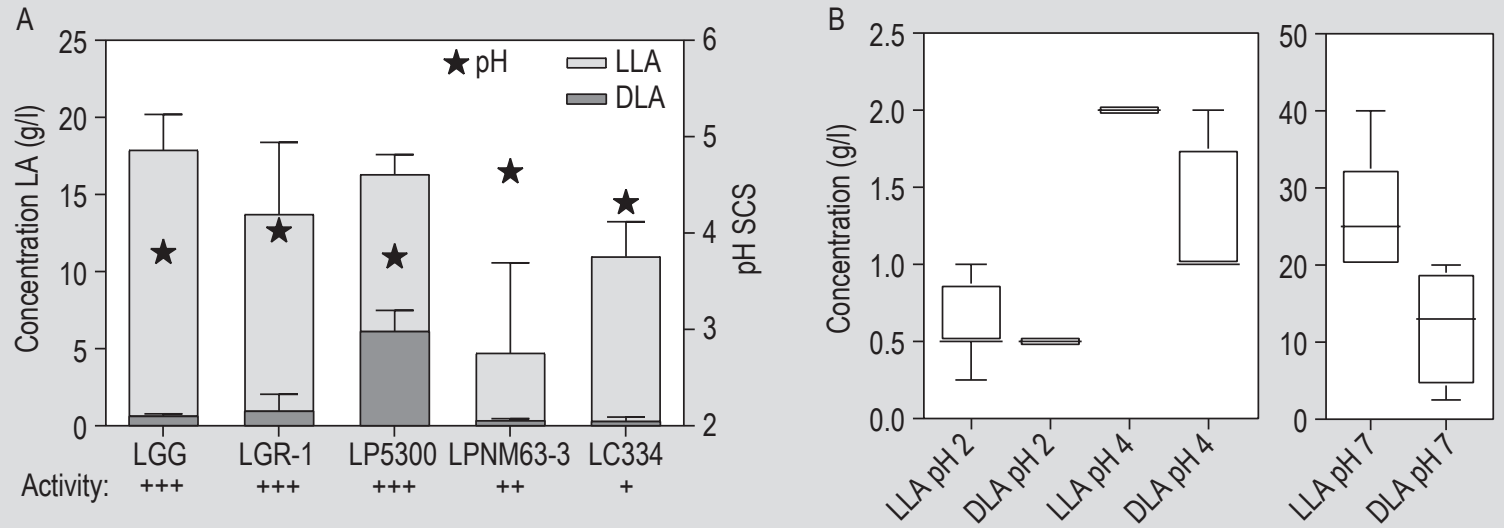

Figure 3. Antimicrobial activity of D- and L-lactic acid (-LA) against Moraxella catarrhalis. (A) Concentration of D- and L-LA produced, $\mathrm{pH}$ and activity of different Lactobacillus strains tested after growth for $19 \mathrm{~h}$ in MRS medium. (B) MIC assay results of LA under different $\mathrm{pH}$ conditions. The median of the results are given with the upper and lower limit of the data between brackets. Names of the Lactobacillus strains can be found in Table 1.

rhamnosus GG in a 1:1 or 10:1 ratio reduced adhesion of M. catarrhalis 1.9 - and 2.6-fold, respectively (adjusted $P$-values both 0.012$)$. Furthermore, a significant 1.8- (46\%),
1.9- (46\%), 1.2- (16\%) and 1.5-fold (33\%) reduction in IL-1 $\beta$, IL-8, MUC5AC and TNF- $\alpha$ gene expression, respectively, was observed upon 1:1 co-incubation with L. rhamnosus 


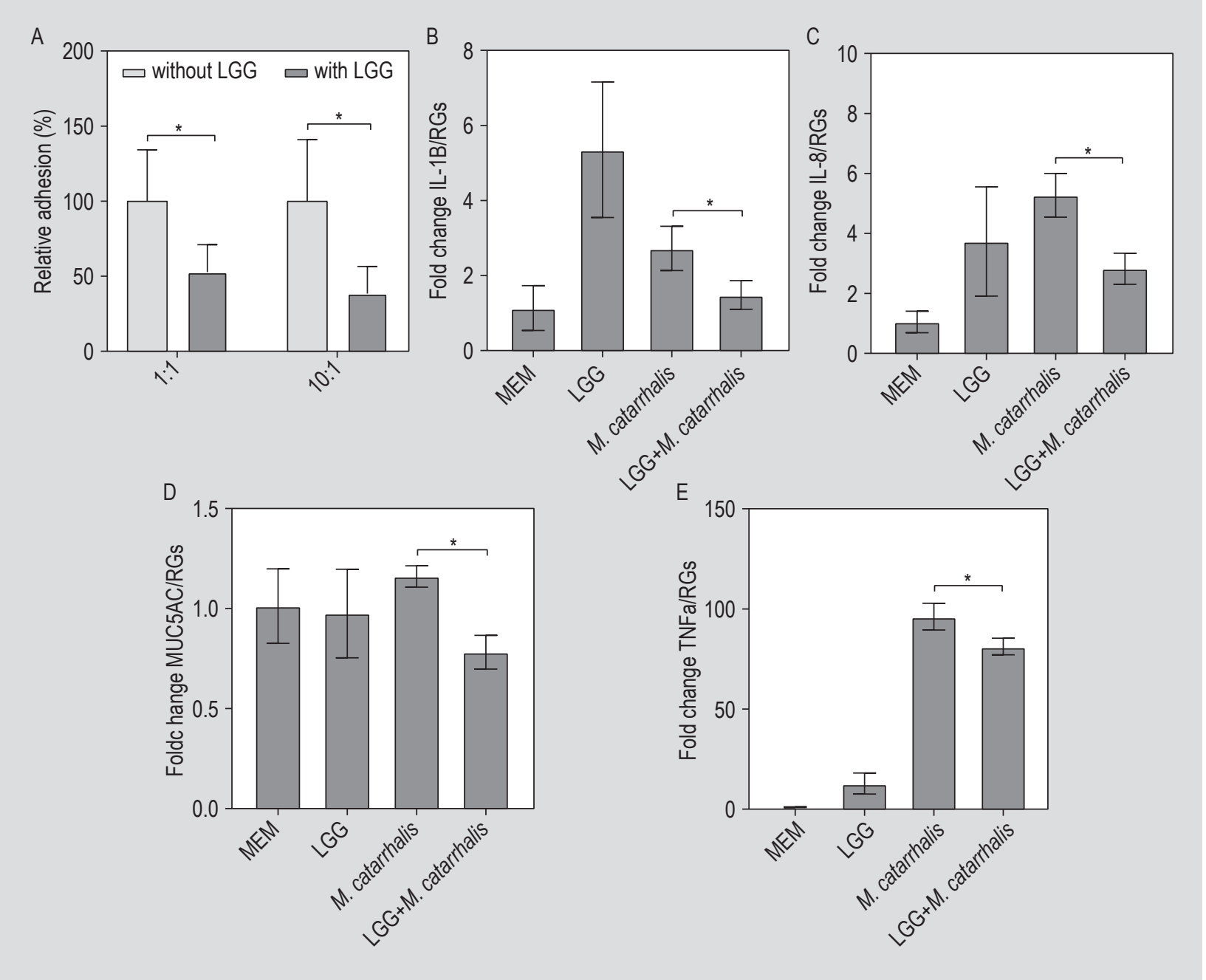

Figure 4. Competition in (A) adhesion and (B-E) immunomodulatory activity of Lactobacillus rhamnosus GG (LGG) vs Moraxella catarrhalis. mRNA expression of (B) interleukin (IL)-1 $\beta$, (C) IL-8, (D) MUC5AC and (E) tumour necrosis factor alpha (TNF- $\alpha$ ). MEM $=$ only MEM medium. ${ }^{*} P<0.05$.

GG compared to $M$. catarrhalis only in the Calu-3 cell line (Adjusted $P$-values: 0.028, 0.027, 0.030 and 0.011, respectively) (Figure 4B-4E).

\section{Discussion}

URT infections mediated by $M$. catarrhalis in adults and children have a high incidence and thus form a major health threat. Furthermore, they form a common reason for antibiotic prescription in the clinical practice. Yet, unnecessary prescribing of antibiotics is costly, leads to serious unintended side effects, and increases the risk of developing antibiotic resistance (Boatright et al., 2015). There is thus a clear need for alternatives for antibiotic therapy and prevention, particularly against Gram-negative pathogens (Wenzler et al., 2016). Since pioneering microbial profiling studies have suggested correlations between the occurrence of LAB and reduced risk of URT infections, the use of probiotic lactobacilli in the fight against URT infections holds promise. In this study, we have shown for the first time - to the best of our knowledge - detailed data supporting a direct antimicrobial role for lactobacilli against a main URT pathogen, M. catarrhalis.

Our results show a clear potential for several Lactobacillus strains to inhibit the growth of $M$. catarrhalis in suspension and as biofilm. Furthermore, L. rhamnosus GG was shown to compete with $M$. catarrhalis for adhesion to human airway epithelial Calu-3 cells and decrease the gene expression of IL-1 $\beta$, IL-8, MUC5AC and TNF- $\alpha$. Both actively growing lactobacilli and their secreted metabolites in SCS showed a clear direct antimicrobial activity against $M$. catarrhalis in agar-based assays. As to the identification of active compounds, acids appeared to play a major role in the inhibition of the pathogen, since the activity disappeared after neutralising the $\mathrm{pH}$. Furthermore, a link was found between the concentration of lactic acid in the SCS and 
the inhibitory activity whichwas also confirmed in brothbased assays.

Most lactobacilli are homofermentative LAB that produce D- and/or L-lactic acid. To investigate the importance of both isomers in the antimicrobial activity of the Lactobacillus species, MIC assays were performed. We observed a MIC varying between 0.5 and $27 \mathrm{~g} / \mathrm{l}$ depending on the $\mathrm{pH}$ for $\mathrm{L}$ - and D-lactic acid. These MIC values are higher than the MIC of $16 \mathrm{mg} / \mathrm{l}$ for amoxicillin, a common used antibiotic for URT infections (Harrison et al., 2009). However, these values should be considered in the context of the human microbiota where live bacteria interact and where applied probiotics could continuously secrete lactic acid as a consequence of their metabolism when alive. Since previous microbiome profiling studies have documented that various Lactobacillus OTUs can be present in several individuals in relative amounts up to $13.29 \%$ in the URT niche (Abreu et al., 2012; Bogaert et al., 2011; Laufer et al., 2011; Stearns et al., 2015), a local production of lactic acid, or antimicrobial compounds in general, could be sufficient to compete in a direct way with the associated pathogens. In this way, a bacteriostatic activity could preserve the microbial balance in this niche and prevent overgrowth of unwanted pathogens. It is important to note that the MIC values of lactic acid against $M$. catarrhalis were found here to significantly increase with increasing $\mathrm{pH}$. As previously ascertained for the pathogens E. coli, Pseudomonas aeruginosa and Salmonella enterica serovar Typhimurium (Alakomi et al., 2005), the undissociated form of lactic acid appears to be most active also against the Gram-negative pathogen M. catarrhalis.

Although we could clearly substantiate an antimicrobial role for lactic acid against $M$. catarrhalis in our present work, possible activity of additional molecules can not be ruled out. These other molecules seem heat-resistant and $\mathrm{pH}$-dependent based on the agar-based antimicrobial and biofilm assays and could have synergistic effects with lactic acid. However keeping in mind that the agar-based assays used in this study favour the identification of small active molecules, due to differences in diffusion velocities as described by Bonev et al. (2008), the involvement of other molecules cannot be ruled out. For example, Niku-Paavola et al. (1999) observed an inhibitory substance, the cyclic molecule mevalonolactone, in the SCS of $L$. plantarum VTT E-78076 which activity increased from 15 to $60 \%$ inhibition in the presence of $1 \%$ lactic acid (activity 40\%). Such synergistic effects are likely secondary to the possible permeabilisation of the membrane by lactic acid. In addition, we could recently demonstrate an antibiofilm activity of lectin-like proteins of L. rhamnosus GG against other Gram-negative pathogens (Petrova et al., 2016) pointing to additional antimicrobial mechanisms of lactobacilli.
More indirect activities such as competition for adhesion sites and nutrients (competitive exclusion) and modulations of the immune system are also postulated to be key factors in the antimicrobial potential of probiotics (Lebeer et al., 2008). In this study, in addition to its direct antimicrobial effect, the effect of $L$. rhamnosus GG on adhesion and pro-inflammatory gene expression in Calu-3 cells by $M$. catarrhalis was therefore investigated. L. rhamnosus GG significantly dampened the gene expression of IL-1 $\beta$, IL-8 and TNF- $\alpha$ after incubation with Moraxella. In addition, the gene expression of MUC5AC, a mucin commonly correlated with OM (Kerschner et al., 2010), was significantly reduced. Especially in the URT, mucin production can be problematic and correlated with pathogens as they benefit from the increased nutrient availability and adherence possibility. Also Abreu et al. (2012) demonstrated in a mouse model that another URT pathogen (Corynebacterium tuberculostearicum) significantly increased the mucin secretion in the host, which could be attenuated in the presence of Lactobacillus sakei. The importance and exact molecular signalling events in the activity of lactobacilli against mucin hypersection remains however to be further explored.

To conclude, we have documented in this study that several Lactobacillus species show antimicrobial activities against M. catarrhalis. Local application of probiotic bacteria could thus have potential as a therapeutic or preventive strategy against URT infections in which this pathogen is involved. However, before such therapies will be feasible, clinical studies in humans will have to confirm our findings.

\section{Acknowledgements}

We would like to thank the Lebeer Lab of Applied Microbiology and Biotechnology (UAntwerp) and Nizet Lab (UCSD) for the technical help and contribution to the manuscript. This research was funded by DOC PRO FFB130135 (UAntwerp), IWT-SBO (Vlaio) ProCure IWT 150052, IOF/SBO FF/130233 (UAntwerp), FWO KaN 6522 and FWO travel grant for a short stay abroad K228515N.

\section{References}

Abreu, N.A., Nagalingam, N.A., Song, Y., Roediger, F.C., Pletcher, S.D., Goldberg, A.N. and Lynch, S.V., 2012. Sinus microbiome diversity depletion and corynebacterium tuberculostearicum enrichment mediates rhinosinusitis. Science Translational Medicine 4: 151ra124.

Alakomi, H., Skyttä, E., Saarela, M. and Helander, I.M., 2005. Lactic acid permeabilizes gram-negative bacteria by disrupting the outer membrane lactic acid permeabilizes gram-negative bacteria by disrupting the outer membrane. Applied and Environmental Microbiology 66: 2000-2005. 
Biesbroek, G., Bosch, A.A.T.M., Wang, X., Keijser, B.J.F., Veenhoven, R.H., Sanders, E.A.M. and Bogaert, D., 2014. The impact of breastfeeding on nasopharyngeal microbial communities in infants. American Journal of Respiratory and Critical Care Medicine American Thoracic Society 190: 298-308.

Boatright, C., Holcomb, L. and Replogle, W., 2015. Treatmnent patterns for pediatric acute CHUs media: a gap in evidence-based theory and clinical practice. Pediatric Nursing 41: 271-276.

Bogaert, D., Keijser, B., Huse, S., Rossen, J., Veenhoven, R., Van Gils, E., Bruin, J., Montijn, R., Bonten, M. and Sanders, E., 2011. Variability and diversity of nasopharyngeal microbiota in children: a metagenomic analysis. PLoS ONE 6: e17035.

Bonev, B., Hooper, J. and Parisot, J., 2008. Principles of assessing bacterial susceptibility to antibiotics using the agar diffusion method. Journal of Antimicrobial Chemotherapy 61: 1295-1301.

Bustin, S.A., Benes, V., Garson, J.A., Hellemans, J., Huggett, J., Kubista, M., Mueller, R., Nolan, T., Pfaffl, M.W., Shipley, G.L., Vandesompele, J. and Wittwer, C.T., 2009. The MIQE guidelines: minimum information for publication of quantitative real-time PCR experiments. Clinical Chemistry 55: 611-622.

Chan, R.C., Bruce, A.W. and Reid, G., 1984. Adherence of cervical, vaginal and distal urethral normal microbial flora to human uroepithelial cells and the inhibition of adherence of gram-negative uropathogens by competitive exclusion. Journal of Urology 131: 596-601.

Chan, R.C.Y., Reid, G., Irvin, R.T., Bruce, A.W. and Costerton, J.W., 1985. Competitive exclusion of uropathogens from human uroepithelial cells by Lactobacillus whole cells and cell wall fragments. Infection and Immunity 47: 84-89.

Danielsen, M., 2002. Characterization of the tetracycline resistance plasmid pMD5057 from Lactobacillus plantarum 5057 reveals a composite structure. Plasmid 48: 98-103.

De Keersmaecker, S.C.J., Verhoeven, T.L.A., Desair, J., Marchal, K., Vanderleyden, J. and Nagy, I., 2006. Strong antimicrobial activity of Lactobacillus rhamnosus GG against Salmonella typhimurium is due to accumulation of lactic acid. FEMS Microbiology Letters 259: 89-96.

Goossens, H., Ferech, M., Vander Stichele, R. and Elseviers, M., 2005. Outpatient antibiotic use in Europe and association with resistance: a cross-national database study. The Lancet 365: 579-587.

Harrison, C.J., Woods, C., Stout, G., Martin, B. and Selvarangan, R., 2009. Susceptibilities of Haemophilus influenzae, Streptococcus pneumoniae, including serotype 19A, and Moraxella catarrhalis paediatric isolates from 2005 to 2007 to commonly used antibiotics. Journal of Antimicrobial Chemotherapy 63: 511-519.

Hatakka, K., Blomgren, K., Pohjavuori, S., Kaijalainen, T., Poussa, T., Leinonen, M., Korpela, R. and Pitkäranta, A., 2007. Treatment of acute otitis media with probiotics in otitis-prone children a double-blind, placebo-controlled randomised study. Clinical Nutrition 26: 314-321.
Kankainen, M., Paulin, L., Tynkkynen, S., Von Ossowski, I., Reunanen, J., Partanen, P., Satokari, R., Vesterlund, S., Hendrickx, A.P.A., Lebeer, S., De Keersmaecker, S.C.J., Vanderleyden, J., Hamalainen, T., Laukkanen, S., Salovuori, N., Ritari, J., Alatalo, E., Korpela, R., Mattila-Sandholm, T., Lassig, A., Hatakka, K., Kinnunen, K.T., Karjalainen, H., Saxelin, M., Laakso, K., Surakka, A., Palva, A., Salusjarvi, T., Auvinen, P. and De Vos, W.M., 2009. Comparative genomic analysis of Lactobacillus rhamnosus GG reveals pili containing a human-mucus binding protein. Proceedings of the National Academy of Sciences of the USA 106: 17193-17198.

Kerschner, J.E., Tripathi, S., Khampang, P. and Papsin, B.C., 2010. $M U C 5 A C$ expression in human middle ear epithelium of patients with otitis media. Archives of Otolaryngology - Head \& Neck Surgery American Medical Association 136: 819.

Kleerebezem, M., Boekhorst, J., Van Kranenburg, R., Molenaar, D., Kuipers, O.P., Leer, R., Tarchini, R., Peters, S.A., Sandbrink, H.M., Fiers, M.W.E.J., Stiekema, W., Lankhorst, R.M.K., Bron, P.A., Hoffer, S.M., Groot, M.N.N., Kerkhoven, R., De Vries, M., Ursing, B., De Vos, W.M. and Siezen, R.J., 2003. Complete genome sequence of Lactobacillus plantarum WCFS1. Proceedings of the National Academy of Sciences of the USA 100: 1990-1995.

Laufer, A.S., Metlay, J.P., Gent, J.F., Fennie, K.P., Kong, Y. and Pettigrewa, M.M., 2011. Microbial communities of the upper respiratory tract and otitis media in children. mBio 2: e00245-10.

Lebeer, S., Vanderleyden, J. and De Keersmaecker, S.C.J., 2008. Genes and molecules of lactobacilli supporting probiotic action. Microbiology and Molecular Biology Reviews 72: 728-764.

Lebeer, S., Verhoeven, T.L.A., Vélez, M.P., Vanderleyden, J. and De Keersmaecker, S.C.J., 2007. Impact of environmental and genetic factors on biofilm formation by the probiotic strain Lactobacillus rhamnosus GG. Applied and Environmental Microbiology 73: 6768-6775.

Lie, V., Hudault, S. and Servin, A.L., 1997. Antibacterial effect of the adhering human. antimicrob. Agents Chemother 41: 1046-1052.

Malik, S., Petrova, M.I., Claes, I.J.J., Verhoeven, T.L.A., Busschaert, P., Vaneechoutte, M., Lievens, B., Lambrichts, I., Siezen, R.J., Balzarini, J., Vanderleyden, J. and Lebeer, S., 2013. The highly autoaggregative and adhesive phenotype of the vaginal Lactobacillus plantarum strain CMPG5300 is sortase dependent. Applied and Environmental Microbiology 79: 4576-4585.

Malik, S., Siezen, R.J., Renckens, B., Vaneechoutte, M., Vanderleyden, J. and Lebeer, S., 2014. Draft genome sequence of Lactobacillus plantarum CMPG5300, a human vaginal isolate. Genome Announcements 2: e01149-14.

Murphy, T.F., Parameswaran, G.I. and Parameswaran, G.I., 2009. Moraxella catarrhalis, a human respiratory tract pathogen. Clinical Infectious Diseases 49: 124-131.

Niittynen, L., Pitkäranta, A. and Korpela, R., 2012. Probiotics and otitis media in children. International Journal of Pediatric Otorhinolaryngology 76: 465-470.

Niku-Paavola, M.L., Laitila, A., Mattila-Sandholm, T. and Haikara, A., 1999. New types of antimicrobial compounds produced by Lactobacillus plantarum. Journal of Applied Microbiology 86: 29-35. 
Nizet, V., Ohtake, T., Lauth, X., Trowbridge, J., Rudisill, J., Dorschner, R.A., Pestonjamasp, V., Piraino, J., Huttner, K. and Gallo, R.L., 2001. Innate antimicrobial peptide protects the skin from invasive bacterial infection. Nature 414: 454-457.

Parameswaran, G.I., Wrona, C.T., Murphy, T.F. and Sethi, S., 2009. Moraxella catarrhalis acquisition, airway inflammation and protease-antiprotease balance in chronic obstructive pulmonary disease. BMC Infectious Diseases 9: 178.

Pearson, M.M. and Hansen, E.J., 2007. Identification of gene products involved in biofilm production by Moraxella catarrhalis ETSU-9 in vitro. Infection and Immunity American Society for Microbiology 75: 4316-4325.

Perez, A.C., Pang, B., King, L.B., Tan, L., Murrah, K.A., Reimche, J.L., Wren, J.T., Richardson, S.H., Ghandi, U. and Swords, W.E., 2014. Residence of Streptococcus pneumoniae and Moraxella catarrhalis within polymicrobial biofilm promotes antibiotic resistance and bacterial persistence in vivo. Pathogens and Disease 70: 280-288.

Petrova, M.I., Imholz, N.C.E., Verhoeven, T.L.A., Balzarini, J., Van Damme, E.J.M., Schols, D., Vanderleyden, J. and Lebeer, S., 2016. Lectin-like molecules of Lactobacillus rhamnosus GG inhibit pathogenic Escherichia coli and Salmonella biofilm formation. PLoS ONE 11: e0161337.

Pettigrew, M.M., Laufer, A.S., Gent, J.F., Kong, Y., Fennie, K.P. and Metlay, J.P., 2012. Upper respiratory tract microbial communities, acute otitis media pathogens, and antibiotic use in healthy and sick children. Applied and Environmental Microbiology 78: 6262-6270.
Rautava, S., Salminen, S. and Isolauri, E., 2009. Specific probiotics in reducing the risk of acute infections in infancy - a randomised, double-blind, placebo-controlled study. British Journal of Nutrition 101: 1722 .

Reid, G. and Bruce, A.W., 2001. Selection of Lactobacillus strains for urogenital probiotic applications. Journal of Infectious Diseases 183: 77-80.

Reid, G. and Reid, G., 1999. The scientific basis for probiotic strains of Lactobacillus. Applied and Environmental Microbiology 65: 3763-3766.

Rovers, M.M., Schilder, A.G., Zielhuis, G.A. and Rosenfeld, R.M., 2004. Otitis media. Lancet 363: 465-473.

Schillinger, U. and Lücke, F.K., 1989. Antibacterial activity of Lactobacillus sake isolated from meat. Applied and Environmental Microbiology 55: 1901-1906.

Stearns, J.C., Davidson, C.J., McKeon, S., Whelan, F.J., Fontes, M.E., Schryvers, A.B., Bowdish, D.M.E., Kellner, J.D. and Surette, M.G., 2015. Culture and molecular-based profiles show shifts in bacterial communities of the upper respiratory tract that occur with age. ISME Journal 9: 1268-1268.

Tagg, J.R. and Dierksen, K.P., 2003. Bacterial replacement therapy: adapting 'germ warfare' to infection prevention. Trends in Biotechnology 21: 217-223.

Wenzler, E., Fraidenburg, D.R., Scardina, T. and Danziger, L.H., 2016. Inhaled antibiotics for gram-negative respiratory infections. Clinical Microbiology Reviews 29: 581-632. 
